\title{
Eva Kowollik
}

\section{Das Leiden der Anderen? Scheitern und Chancen dialogischen Erinnerns in Goran Vojnovićs Jugoslavija, moja dežela}

\begin{abstract}
Ne spadam, međutim, među one koji misle da se sve to može oprostiti ili zaboraviti i krenuti dalje. Po tom sam pitanju vrlo radikalan i imam čvrst stav kao čovjek: dok žive žrtve, žive $i$ njihovi zločinci i zločini. (Vojnović 2013b)
\end{abstract}

Ich gehöre allerdings nicht zu denjenigen, die denken, dass man das alles verzeihen oder vergessen und weitermachen kann. In dieser Frage bin ich sehr radikal und habe als Mensch einen festen Standpunkt: solange die Opfer leben, leben auch die Verbrecher und Verbrechen. ${ }^{1}$

In der folgenden Analyse des Status von (nationalen) Opfernarrativen in einem paradigmatischen Text der postjugoslawischen Literaturszene möchte ich die Anwendbarkeit von Aleida Assmanns Konzept des „dialogischen Erinnerns“ auf den postjugoslawischen literarischen Diskurs prüfen: „Zwei Staaten entwickeln ein dialogisches Erinnerungsmodell, wenn sie einseitig oder gegenseitig ihren eigenen Anteil an der traumatisierten Geschichte des anderen anerkennen und empathisch das selbst verursachte und zu verantwortende Leiden der anderen Nation ins eigene Gedächtnis mit einschließen“ (2016, 195-196). Das Besondere an Assmanns dialogischem Erinnern gegenüber vergleichbaren Erinnerungskonzepten, die ebenfalls das Moment der Verschränkung favorisieren, ${ }^{2}$ liegt einerseits in seiner breiten Anwendbarkeit auf vielfältige transnationale Kontexte, und andererseits in der Tatsache, dass der Prozess der Verknüpfung von Erinnerungen als Dialog gedacht ist, das heißt als sprachlicher Akt, der auf eine bestimmte

1 Sofern nicht anders vermerkt, stammen die Übersetzungen von der Verfasserin - E. K.

2 Von Ansätzen des kommunikativen Gedächtnisses (vgl. z.B. Welzer 2002) unterscheidet sich Assmanns dialogischer Ansatz durch die Ausweitung auf Kommunikationssituationen in politischen Kontexten. Ebenfalls in eine transnationale Richtung geht Michael Rothbergs Konzept der multidirectional memory (2009), angewandt auf die Verschränkung der Shoah mit kolonialem Erinnern. Im Kontext der postjugoslawischen Literaturen verwendet Stijn Vervaet anhand von Texten, die Shoahnarrative mit dem Erzählen der jugoslawischen Zerfallskriege verknüpfen, den Begriff der intersecting memories (2016). 
Konstellation aus Sprecher^innen und Zuhörer*innen hindeutet. Lassen sich in den jugoslawischen Nachfolgestaaten, insbesondere im Hinblick auf die jugoslawischen Zerfallskriege, dialogische Muster des Erinnerns identifizieren?

\section{Kontextualisierungen}

Eine Erinnerungskultur im Assmann'schen Sinne hat in den postjugoslawischen Gesellschaften (noch) keine breite gesellschaftliche Basis. ${ }^{3}$ Dass die nationalen Erinnerungsdiskurse weithin dem Modell des eigenen Leidens verhaftet sind, hängt mit der problematischen Erinnerungsdoktrin im jugoslawischen Staatssozialismus zusammen, die zudem auf den Partisanenmythos und die Losung Bratstvo i jedinstvo (Brüderlichkeit und Einheit) setzte:

The official interpretation of the war contained two principle aspects: first, it relied on a simple dichotomy, presenting the Partisans as ,revolutionaries‘ and ,liberators' and all other forces as ,counterrevolutionaries' and ,fascists'; secondly, it effectively ,de-ethnicised ‘ the war, by blaming the ,bourgeoisies‘ of all Yugoslav nations for the crimes that had taken place and by dealing with wartime inter-ethnic conflicts only in terms of superficial reciprocity [...]. (Dragović-Soso 2002, 100)

Als Folge dieses Simplifizierungsgebotes schwelten somit im zweiten Jugoslawien auf allen Seiten nicht verarbeitete historische Traumata im äußerst flexiblen Familiengedächtnis weiter - eine von Ressentiments begleitete transgenerationale Weitergabe blieb der einzige Weg des Erinnerns. ${ }^{4}$ In der Literatur kulminierte dies in äußerst populären Bestsellern, die seit den 1980er Jahren auf dem Buchmarkt erschienen und einer "Literatur der populistischen Welle“ angehörten. ${ }^{5}$ Charakterisiert waren diese Texte von einer selektiven Wahrnehmung ausschließlich des eigenen Leidens und einer Identifikation mit der eigenen Opferposition in der Vergangenheit, ein Phänomen, das Alida Bremer 1992 in ihrer Diagnose der damals „neueren serbischen Literatur“ treffend als „eine Art kollektiven inneren Monolog“ (1992, 460) bezeichnete.

3 In ihrer Einleitung weist Assmann mit den Fragen „Warum gab es nach dem Ende des Zweiten Weltkriegs keine ,Erinnerungskultur'? Warum galt lange Zeit das Schweigen als die bessere Option?“ (2016, 10) darauf hin, dass für die Entwicklung einer Erinnerungskultur im Zusammenhang mit kollektiven Traumata eine gewisse zeitliche Distanz notwendig ist.

4 Zur Flexibilität des sozialen Gedächtnisses vgl. Assmann 2014, 203.

5 Der Begriff stammt von Mirko Djordjević (1998). 
Nun stellt sich im Gegenzug die Frage, welchen Stellenwert das „Leiden der Anderen“6 in einem alternativen literarischen Diskurs einnimmt, der sich nationalkultureller Vereinnahmung widersetzt und sich dezidiert als ,postjugoslawisch` verstanden wissen will.

Unter postjugoslawischer Literatur ${ }^{7}$ verstehe ich einerseits „literature, which is ultimately informed by, but also critical of Yugoslav ideology“ (Matijević 2016, 107), andererseits ist es „literature, which is post-Yugoslav in its critique of post-Yugoslav nationalisms“ (Matijević 2016, 107). Die Bezeichnung ließe sich also dahingehend interpretieren, dass das Präfix ,post‘ sowohl die Notwendigkeit impliziert, zurückzuschauen, als auch, die Vergangenheit zu überwinden (Matijević 2016, 107-108). Diese Argumentation weiterverfolgend meint postjugoslawische Literatur also „the space of articulation of dissident and alternative cultural and political voices“, sie verfüge über „capacity of resistance“ (Matijević 2016, 102) oder sei als emanzipatorischer Diskurs (Matijević 2016, 102) ${ }^{8}$ beschreibbar. Es wäre also zu prüfen, inwieweit sich der Begriff des dialogischen Gedächtnisses für die Beschreibung thematischer und struktureller Charakteristika postjugoslawischer literarischer Texte eignet, sowie in literatursoziologischer Hinsicht für Prozesse und Wirkungen von Texten und ihren Akteur`innen im Feld der postjugoslawischen Literaturen.

Eine empathische Orientierung am „Leiden der Anderen“, mithin „Opferorientierung“ “, ${ }^{9}$ beschränkt sich in den postjugoslawischen Gesellschaften auf die einer widerständigen Subkultur angehörende, sich nationalkulturellen Diskursen widersetzende, alternative postjugoslawische Kulturszene. ${ }^{10}$

6 Mit diesem Syntagma stütze ich mich auf den Text des Literaturwissenschaftlers Davor Beganović: „Poštovanje drugoga, patnja drugoga“ (2015) [Die Achtung des Anderen, das Leiden des Anderen], wobei „das Leiden des Anderen“ das „ethische Potenzial“ (378) postjugoslawischer Literatur umschreiben soll.

7 Meine Darstellung des postjugoslawischen literarischen Diskurses folgt der Argumentation von Tijana Matijević (2016). Ihr Text reagiert auf die kritische Frage nach dem ,post‘ im Begriff ,postjugoslawisch', die Boris Postnikov aufwirft (Boris Postnikov. Postjugoslavenska književnost? Zagreb: Sandorf 2012, zitiert nach Matijević 2016, 103-105).

8 Matijević stützt sich mit dieser letzten Einschätzung auf Svetlana Slapšaks Text „Twin Cultures and Rubik's Cube Politics: The dynamics of Cultural Production in Pro-YU, Post-YU, and Other YU Inventions“. Südosteuropa: Zeitschrift für Politik und Gesellschaft 3 (2011): 301-314, 311. Zitiert nach Matijević 2016, 102.

9 Assmann verweist auf die sinnvolle Differenzierung in „opferidentifiziert“ und „opferorientiert“, die Werner Konitzer (2012) vorschlägt.

$10 \mathrm{Zu}$ dieser zählen selbstverständlich auch prominente, oft im westlichen Ausland präsente und von dort aus protegierte Vertreter^innen, wie Dubravka Ugrešić, David Albahari oder Miljenko Jergović. 
Aus diesen Kontextualisierungsversuchen heraus gilt es, für die Textanalyse zwei Ebenen zu unterscheiden. Einerseits ist die Ebene der postjugoslawischen Literatur als ein literarisches Feld $\mathrm{zu}$ betrachten, in dem dialogisches Erinnern als Intention (bezogen auf den Prozess der Literaturproduktion) und als ein Wirkpotenzial (bezogen auf den Prozess der Rezeption) identifiziert werden könnte, ohne die damit verbundenen Schwierigkeiten auf den Literaturmärkten der Region vernachlässigen $\mathrm{zu}$ wollen. Im Vordergrund sollen jedoch die im literarischen Text inszenierten Erinnerungsprozesse stehen, die wiederum eher auf eine Verhärtung im Erinnerungsdiskurs in der Region hinweisen. Als Katalysator für das Misslingen dialogischen Erinnerns auf der Ebene der Figurenkonstellation fungiert nun im hier fokussierten Roman des slowenischen Autors Goran Vojnović Jugoslavija, moja dežela (2011, dt. Vaters Land, 2016) ein äußerst lebendiges Opfernarrativ.

\section{Opferkonkurrenzen, Opfernarrativ und dialogisches Erinnern}

Wie oben skizziert, ist in den postjugoslawischen Gesellschaften - und nicht nur dort - dialogisches Erinnern nicht die Regel, sondern die Ausnahme. Geprägt ist der Erinnerungsdiskurs sowohl auf sozialer als auch auf der Ebene der jeweiligen Nationen von Opferkonkurrenzen, Opferhierarchien und Selbstviktimisierung. Ursache dafür sind Phänomene, die Aleida Assmann treffend als „Asymmetrie zwischen Opfer- und Tätergedächtnis“ $(2014,82)$ beschreibt. So könne für das Tätergedächtnis eine Tendenz zur „Selbstviktimisierung“ konstatiert werden, eine „semantische Umkodierung [...], eine Haltung, die das eigene Leiden in den Mittelpunkt stellt und dabei gleichzeitig von der eigenen Verstrickung in die Unrechtsgeschichte ablenkt“ (Assmann 2016, 145). ${ }^{11}$ Im Zusammenhang mit Selbstviktimisierung und einer „Rhetorik der Aufrechnung“ (Assmann 2014, 170) ist der Einfluss von Institutionen und politischen oder intellektuellen Akteur*innen „zur Konstitution von Erinnerungs- und Erzählmustern“ (Schulze Wessel 2012, 3) nicht zu unterschätzen, worauf Martin Schulze Wessel in der Einleitung zum Band Opfernarrative hinweist.

Für den serbischen Kontext zeigt die Politikwissenschaftlerin Jasna DragovićSoso in ihrer Studie, Saviours of the Nation': Serbia's Intellectual Opposition and

11 Assmann bezieht sich mit dem Begriff „Selbstviktimisierung“ auf den Historiker Martin Sabrow, der diese Erscheinung in der deutschen Erinnerungspolitik beschreibt (Sabrow 2012). 
the Revival of Nationalism (2002), wie mittels literarischer Texte, aber auch medialer Einflussnahme und wissenschaftlicher Stellungnahmen ${ }^{12}$ die Geschichte des Zweiten Weltkrieges umgewertet und der Mythos der Opferrolle des serbischen Volkes gestärkt wurde. Die Verfolgung der Serben durch die Ustaša wurde ,the most potent historical symbol of Serbian victimisation in the latter part of the 1980s“ (Dragović-Soso 2003, 103) und fand Eingang in den zu diesem Zeitpunkt auch öffentlich artikulierbaren serbischen Erinnerungsdiskurs. ${ }^{13}$ Die nationalen „Erinnerungsrahmen“"14 schaffen seitdem - und nicht nur in Serbien - feste Strukturen für Opfernarrative und forcieren damit die Erinnerung an die jeweils eigenen Leiden im Zweiten Weltkrieg sowie während der jugoslawischen Zerfallskriege. Dies betrifft alle Seiten: Wolfgang Höpken konstatierte 2006 exemplarisch für die slowenische und kroatische Seite der postjugoslawischen Erinnerungskulturen in Bezug auf traumatische Erinnerungsorte aus dem Zweiten Weltkrieg: „Das Bemühen, auch zu einer konsensfähigen Narration der Kriegsgeschichte zu kommen, steht allerdings noch aus“ (2006, 422). Daniela Mehler fordert - hier mit Blick auf die serbische Gesellschaft - „einen gesellschaftlichen Dialog [...], der sich kritisch und produktiv mit der Vergangenheit auseinandersetzt, auch Graustufen, auch die Doppelrollen von Akteuren als Täter-Opfer sowie OpferTäter und Sprecher mit einer anderen Position zulässt“ (2015, 298-299). Gefordert ist also ein in den gesellschaftlichen Strukturen verankerter dialogischer Erinnerungsdiskurs.

Aleida Assmann hat allerdings mit Bezug auf den negativen Klang des Wortes „Selbstviktimisierung“ zu Recht auf die Gefahr der Etikettierung von Leidensgeschichten aufmerksam gemacht: „Dieses Denken bleibt von einer AusschlussLogik der gegenseitigen Verdrängung bestimmt, die den Erinnerungsdiskurs weiterhin beherrscht. Sie unterstellt, dass die Erinnerung an die eigenen Leiden

$12 \mathrm{Zu}$ den bekanntesten und folgenschwersten dieser Stellungnahmen gehört zweifellos das Memorandum der Serbischen Akademie der Wissenschaften (Memorandum SANU); vgl. dazu auch Olivera Milosavljevićs Text Der Mißbrauch der Autorität der Wissenschaft (1998).

13 Den Umschwung in der serbischen Historiografie beschreibt die serbische Historikerin Ljubinka Trgovčević als Deutung eines ,jahrhundertelangen Völkermordes an den Serben“ (2002, 403); der Historiker Holm Sundhaussen spricht von der „Weiterentwicklung eines nationalen Opfersyndroms, das zum prägenden Signum der Geschichte seit dem ausgehenden vierzehnten Jahrhundert gestaltet wurde“ (2002, 411). Zur Zusammenfassung des historiografischen Diskurses in Serbien ab den 1980er Jahren vgl. das Kapitel „Von der jugoslavischen zur serbischen Nationalgeschichte“ in Kowollik 2013, 18-25.

14 Die Existenz gesellschaftlicher „Erinnerungsrahmen“ versteht Aleida Assmann als notwendige Bedingung für die Wirksamkeit der Artikulation von Traumata, um das Trauma „zu einem Teil der bewussten Identität zu machen“ (2014, 94). 
automatisch ein Bewusstsein für die Leiden der Anderen auslöscht“ $(2016,153)$. Weiterhin spricht sie vom „Recht auf unterschiedliche Erinnerungen“ (2016, 153) und fordert, es dem Menschen zuzutrauen, „dass er über dem eigenen Leid Fragen der Gerechtigkeit und das Leid der Anderen nicht aus dem Auge verliert“ (2016, 154).

Nun ließe sich fragen, welche Bedingungen ein solches dialogisches Erinnern unter Menschen fördern oder behindern. Mit Hilfe von traumatheoretischen Ansätzen, die aus der psychotherapeutisch orientierten Forschung zur Shoah kommen, lassen sich kommunikative Akte bzw. deren Gelingen oder Misslingen als Basis für dialogisches Erinnern identifizieren. Eine sinnstiftende Auseinandersetzung mit Traumata ist zunächst nicht ohne Weiteres möglich, da die präzise Wiederkehr traumatischen Erlebens kein Wissen sei, worüber Traumatisierte aktiv verfügen würden (Caruth 1995). Dem kann eine aktive Form des Zuhörens Abhilfe schaffen, die auf der Bereitschaft der Zuhörenden aufbaut, die Verantwortung für die Vergangenheit mitzutragen. An diesem ebenfalls aus der Psychotherapie stammenden Konzept der „sekundären Zeugenschaft“15 könnte ein dialogisches Erinnern ansetzen.

Wie oben erläutert, weist die postjugoslawische Literatur als „space of articulation of dissident and alternative cultural and political voices“ (Matijević 2016, 102) eine besondere Affinität zu dialogischem Erinnern auf verschiedenen Ebenen auf. Es ist also sinnvoll, Traumaerzählungen der postjugoslawischen Literaturen auf Inszenierungen dialogischer Erinnerungsstrukturen hin zu untersuchen und hierbei die Interaktion von Zuhörer`innen und Erzähler*innen im Hinblick auf konkurrierende Opferdiskurse herauszustellen.

An Goran Vojnovićs Roman Jugoslavija, moja dežela interessiert mich somit auch die Ambivalenz von Opfer-Täter-Konstellationen, die der Text aufwirft und die durch eine transgenerationale Perspektive zustande kommt. Des Weiteren zeigt der Roman auch Grenzen des eben skizzierten Konzepts der Zuhörerschaft und damit dialogischen Erinnerns auf. Schließlich sollen auf der literatursoziologischen Ebene die Chancen des Buches, im Rahmen der postjugoslawischen Literaturszene zu einem dialogischen Erinnern beizutragen, abgeschätzt werden.

15 Vgl. den Band „Niemand zeugt für den Zeugen“: Erinnerungskultur und historische Verantwortung nach der Shoah (2000), herausgegeben von Ulrich Baer. 


\section{Jugoslavija, moja dežela - Trauma und Zuhören}

Jugoslavija, moja dežela, der zweite Roman des slowenischen Autors Goran Vojnović, 2011 in Ljubljana erschienen, ist für eine Untersuchung des Potenzials dialogischen Erinnerns in postjugoslawischen literarischen Texten besonders aufschlussreich, denn Vojnović gilt unter den jüngeren slowenischen Schriftsteller`innen zunächst als derjenige, bei dem die jugoslawischen Zerfallskriege „am prominentesten dargestellt“ (Čeh Steger 2013, 208) ${ }^{16}$ werden; er positioniert sich weiterhin selbst dezidiert als slowenischer Autor innerhalb der postjugoslawischen Literaturszene (Vojnović 2013b) und wird als solcher, wie die Rezeption in der Region beweist, auch im gesamten postjugoslawischen Kulturraum wahrgenommen. Von der Zugehörigkeit zum postjugoslawischen Kulturraum zeugen seine Texte nicht nur in thematischer Hinsicht, sondern auch sprachlich durch eine figurenspezifische Mehrsprachigkeit, durch die sich insbesondere sein erster Roman Čefurji raus! (2008, Südländer raus!) auszeichnet, die aber auch in Jugoslavija, moja dežela anklingt und in seinem jüngsten Roman Figa (2016, dt. Unter dem Feigenbaum) wieder zunimmt. ${ }^{17}$

Worum geht es im Roman? Der Ich-Erzähler Vladan Borojević, in der Erzählgegenwart ein gescheiterter, in Ljubljana lebender Student, stammt aus einer slowenisch-serbischen Familie, die vor den Kriegen an der kroatischen Küste lebte. Mit Kriegsausbruch 1991 wurde Vladan unvermittelt aus einer sorgenfreien Kindheit gerissen. Der Vater Nedeljko, Offizier der Jugoslawischen Volksarmee, wird nach Belgrad beordert und von dort an die Front in Slawonien geschickt. Seine Mutter Duša flieht mit Vladan aus Serbien, wo beide bei Nedeljkos Verwandten untergekommen waren, zurück nach Slowenien. Dort erfährt Vladan von seiner Mutter, sein Vater sei im Krieg gefallen. Das Trauma des Kindes, das mit elf Jahren sein Land, seine Sprache und seinen Vater verloren hat, äußert sich sechzehn Jahre später in deutlichen Symptomen. Vladan erfährt zu diesem Zeitpunkt zufällig bei Recherchen im Internet, dass sein Vater nicht tot ist, sondern als Kriegsverbrecher gesucht wird, verantwortlich für ein Massaker in einem kroatischen Dorf. Die überstürzte Suche nach dem Vater führt ihn nach Kroatien, Bosnien

16 Demgegenüber nimmt laut Čeh Steger die Fiktionalisierung des Zweiten Weltkrieges in Texten der slowenischen Gegenwartsliteratur breiten Raum ein $(2013,200)$.

17 Eine problematische Seite des aktuellen postjugoslawischen literarischen Diskurses stellen getrennte Literaturübersetzungen in Bosnien-Herzegowina, Kroatien und Serbien dar, was sich an Vojnovićs Büchern gut illustrieren lässt. Von Čefurji raus! gibt es eine kroatische, eine serbische und eine bosnische Übersetzung, von Jugoslavija, moja dežela eine kroatische und eine serbische Übersetzung, ebenso von Figa. Für die im postjugoslawischen Literaturfeld kursierenden Texte gibt es also für den Sprachraum des BKMS keine gemeinsame Übersetzerlandschaft. 
und Serbien und wieder zurück nach Slowenien - letztlich findet er ihn in Österreich. Dabei kommt Vladan mit ganz unterschiedlichen Menschen in Kontakt, die seinen Vater gekannt hatten. Schließlich gelingt ihm erst durch Anhören zum Teil absurder Erklärungen, die den Kriegsverbrecher entlasten sollen und auf einem Opfernarrativ aufbauen, die Konfrontation mit dem eigenen Trauma. Auf diese spezifischen Erzählstrukturen soll im Folgenden näher eingegangen werden. Wichtig ist an dieser Stelle noch Folgendes: Goran Vojnović hat mit Jugoslavija, moja dežela keinen autofiktionalen Roman geschrieben, auch wenn autobiografische Details rekonstruierbar sind (Vojnović 2013b). Das Buch zeigt damit einmal mehr die Verflochtenheit auch Sloweniens in die transkulturelle Erinnerungslandschaft im postjugoslawischen Kulturraum, ein Standpunkt, der in Slowenien nicht selbstverständlich ist und worauf Vojnović bereits mit dem Titel des Romans ironisch anspielt. ${ }^{18}$

Im Zentrum steht Vladans Erzählung aus der Ich-Perspektive, angesiedelt im Jahr 2007. Dass es sich beim ersten Kapitel, der letzte unbeschwerte Tag der Pulaer Kindheit im Frühjahr 1991, um eine nicht intendierte Erinnerung des Protagonisten handelt, verdeutlicht erst das zweite Kapitel. Denn das im ersten Kapitel nur nebenbei erwähnte Graffito „KOSOVO REPUBLIKA - ISTRA KONTINENT“ - zwei Jahre lang soll dieses „velikanski grafit“ (Vojnović 2013a, 14) [,riesige[] Graffito“ (Vojnović 2016, 10)] dort bereits stehen - verweist nicht nur deutlich auf bereits anhaltende separatistische Tendenzen seit Ende der 1980er Jahre. Es folgt als ein Flashback unmittelbar auf die Entdeckung des Protagonisten im Internet, dass sein Vater als Kriegsverbrecher gesucht wird, und ist im Buch entsprechend grafisch markiert.

\footnotetext{
Pojma nisem imel, od kod se je dobrih šestnajst let kasneje vzel ta davno pozabljeni pulski grafit [...]. K-O-S-O-V-O-R-E-P-U-B-L-I-K-A-I-S-T-R-A-K-O-N-T-I-N-E-N-T! [...] Moj do nedavna pokojni oče me je skoraj šestnajst let po svoji smrti iz zasede tako neusmiljeno napadel s svojo nesmrtnostjo, da sem lahko skoraj fizično občutil, kako se v meni razrašča občutek groze [...]. (Vojnović 2013a, 17-18) ${ }^{19}$
}

Ich hatte keine Ahnung, woher gute sechzehn Jahre später plötzlich dieses längst vergessene Pulaner Graffito kam [...]. K-O-S-O-V-O-R-E-P-U-B-L-I-K-A-I-S-T-R-A-K-O-N-T-I-N-E-N-T! [...] Mein bis vor Kurzem verstorbener Vater hatte mich fast sechzehn Jahre nach seinem Tod so unbarmherzig mit seiner Unsterblichkeit aus dem Hinterhalt überfallen, dass ich fast physisch spüren konnte, wie in mir das Gefühl des Grauens zunahm [...]. (Vojnović 2016, 12-13)

18 Jugoslavija, moja dežela (wörtlich: Jugoslawien, meine Heimat) ist ein Spiel mit dem Slogan „Slovenija, moja dežela“ [Slowenien, meine Heimat], der in den 1980er Jahren in Slowenien populär geworden war.

19 Zitiert wird der Originaltext aus der 2013 bei Študentska založba erschienenen Ausgabe. 
Der auf der Suche nach dem Vater vorrangig zuhörende Vladan findet, zurück in Slowenien, auch ein zuhörendes Gegenüber - seine Freundin Nadja: „To noč sem premagal molk, [...]. Nadja pa me je le poslušala“ (Vojnović 2013a, 163) [„In dieser Nacht besiegte ich das Schweigen, [...]. Nadja hörte mir nur zu“ (Vojnović 2016, 144-145)]. Nadjas Präsenz wird zu einem entscheidenden, die Handlung motivierenden Moment: Vater und Sohn sollen einander schließlich in Wien treffen, Vladan jedoch ist unmittelbar vor dem Wiedersehen mit seinem Vater Panikattacken ausgesetzt. Durch Nadjas bedingungsloses Da-Sein und Zuhören kann sich der Protagonist überhaupt erst zum Treffen mit seinem Vater aufraffen. Die Inszenierung dieser zwischenmenschlichen Konstellation von Erzählerund Zuhörerschaft in Verbindung mit der Verbalisierung eines Traumas wird im Roman jedoch in all der ihr inhärenten Problematik gezeigt. Die von Vojnović konzipierte Figur der Zuhörerin ist eben keine distanzierte Therapeutin, sondern mit dem Protagonisten liiert, von dem sie sich am Ende trennen wird.

Von dieser Erzähler-Zuhörerin-Konstellation nun sind die Binnenerzählungen zu unterscheiden, denen der Ich-Erzähler während der Reisestationen nun seinerseits zuhört. Diese sollen im Hinblick auf das Opfernarrativ einer genaueren Analyse unterzogen werden.

\section{Multiperspektivische Darstellung des Opfernarrativs}

In einem Interview für die kroatische Zeitung Jutarnji list äußerte Vojnović: „Prokletstvo ratova naših djedova i očeva uvijek nas prati“ (Vojnović 2013b) [Der Fluch der Kriege unserer Großväter und Väter verfolgt uns immer]. Der Autor kommentiert hier die oben bereits skizzierte Problematik der inoffiziellen transgenerationalen Weitergabe jeweils eigener Leidensgeschichten aus dem Zweiten Weltkrieg im sozialistischen Jugoslawien und ihre Auswirkungen bis in die dritte Generation. Wie äußert sich dieses Opfernarrativ nun im Roman?

Vladans erste Station ist Brčko in Bosnien, der letzte seiner Mutter bekannte Aufenthaltsort des Vaters, der zu diesem Zeitpunkt bereits untergetaucht war. Dessen damalige Nachbarin lernte ihn unter dem falschen kroatischen Namen Tomislav Zdravković kennen. ${ }^{20}$ Die Nachbarin erzählt Vladan also des Vaters umerzählte und kroatisierte Geschichte: „Rad je govoril o vseh vas, ampak o tebi

20 Anspielung auf den jugoslawischen Sänger Toma Zdravković, für den der Vater geschwärmt hatte. 
pa največ. Najbrž zato, ker je vedel, da si edini preživel“ (Vojnović 2013a, 58) [„Er hat gern von euch allen erzählt, aber von dir am meisten. Wahrscheinlich weil er wusste, dass du als Einziger überlebt hast“ (Vojnović 2016, 51)]. Nolens volens und durchaus ironisch in der literarischen Darstellung wird das Einzelkind Vladan also zum einzigen Überlebenden, umgeben von ermordeten Geschwistern. Damit wird er Teil eines familiären Opfernarrativs, das, wie Vladan im weiteren Verlauf erfahren wird, eigentlich die Geschichte seines serbischen Großvaters ist, der seine ganze Familie während des Zweiten Weltkrieges in einem Massaker der kroatischen Ustaša verloren hatte.

Einen Hinweis darauf bekommt Vladan bei Emir Muzirović, einst wie Vladans Vater Offizier der Jugoslawischen Volksarmee in Pula. Zum Zeitpunkt von Vladans Besuch lebt Emir zurückgezogen in Goražde, im föderativen Teil Bosniens. Als einstiger Angehöriger der Jugoslawischen Volksarmee sieht Emir in familiär überlieferten Opfererzählungen, in denen Verbrechen aus dem Zweiten Weltkrieg erinnert wurden, und daraus herrührend im Motiv der Rache die Ursache der Verbrechen im neuen Krieg:

O nekih svojih babicah in dedkih, o jamah, o taboriščih. Zgodbo, ki je vse njih vsa ta leta najedala [...]. V bistvu oni sploh niso bili ti, ki so ubijali, ampak so ubijali grobovi njihovih očetov in mater in bratov in sester, [...]. Ker v imenu grobov je vse sveto ... $i$ sve je potrebno ... (Vojnović 2013a, 86-87) ${ }^{21}$

Von Omas und Opas, von Gruben und Lagern. Eine Geschichte, die all die Jahre an ihnen gefressen hatte [...]. In Wirklichkeit waren sie überhaupt nicht die gewesen, die getötet hatten, sondern getötet hatten die Gräber ihrer Väter und Mütter und Brüder und Schwestern, [...]. Denn im Namen der Gräber ist alles heilig... und alles notwendig... (Vojnović 2016, 76-77)

Vladans Vater Nedeljko sei, so Emir, durch das Trauma seines Vaters Milutin im Zweiten Weltkrieg von einer solchen Opfergeschichte geprägt worden. Emir weigert sich, sie Vladan zu erzählen - immerhin habe er, Emir, selbst eine, über die er ja schließlich auch nicht rede.

Emir fühlt sich als ehemaliger General der Jugoslawischen Volksarmee weiterhin gefangen in der tabuisierenden Erinnerungspolitik, die in Titos Jugoslawien bezüglich des Zweiten Weltkrieges galt. Seine nebulöse Redeweise sowie der Respekt ihm gegenüber in Goražde werfen Fragen nach seiner eigenen Ver-

21 Das Zitat ist ein Beispiel, wie Vojnović Mehrsprachigkeit in der Verwendung kursiv markierter bosnischer/kroatischer/serbischer Versatzstücke im slowenischen Text der Figurenrede realisiert. 
gangenheit auf. Er und Vladans Vater kämpften in den Zerfallskriegen zwar offensichtlich auf verschiedenen Seiten, waren aber verbunden durch ihre gemeinsame und als glücklich beschriebene Zeit bei der JNA. Auf diese Weise können sich beide nachträglich als Opfer des von Nationalisten verursachten Zerfalls Jugoslawiens stilisieren und Emir deckt den serbischen Kriegsverbrecher, der ihn vor Jahrzehnten vor einem Mord aus Affekt bewahrt hatte und in dessen Schuld er daher zu stehen glaubt.

Vladans dritte Station ist Novi Sad in Serbien, wo Onkel Danilo lebt, ein Verwandter seines Vaters, bei dem Vladan mit seiner Mutter 1991 vorübergehend lebte. Danilo steht in seinem körperlichen Verfall in einem auffälligen Gegensatz zum Pathos seiner Rede: „s svojo usihajočo pojavo je bil na trenutke v popolnem neskladju s svojimi stopnjujoče nastrojenimi besedami“ (Vojnović 2013a, 114) [„mit seiner versiegenden Erscheinung stand er für Momente in einem völligen Missverhältnis zu seinen auf Steigerung gestimmten Worten“ (Vojnović 2016, 101)]. Dieser (ironisch erzählte) Kontrast in der Figurenkonzeption verbindet übrigens die meisten der Binnenerzähler. Danilo erzählt Vladan nun tatsächlich die Geschichte von Großvater Milutin, eingebettet jedoch in einen generalisierenden, das konkrete Verbrechen relativierenden Opferbegriff:

Tudi sam veš, da ni vojne brez žrtev. Poleg tega pa ... V tisti njegovi vojski je mogoče bilo tudi nekaj pravih vojakov, ampak večinoma so bili to navadni ljudje, ki so jim pred tem pobili družine, požgali hiše. Obupani reveži so bili to, jadnici bez igde ikoga. In daj mi zdaj ti povej, kako naj takšnim ljudem prepoveš, da se maščujejo. (Vojnović 2013a, 112)

Du weißt auch selbst, dass es keinen Krieg gibt ohne Opfer. Und außerdem ... In seiner Armee gab es möglicherweise auch ein paar richtige Soldaten, aber die meisten von ihnen waren gewöhnliche Leute, denen man vorher die Familien umgebracht, die Häuser angezündet hatte. Elende Verzweifelte waren das, arme Kerle ohne irgendwen irgendwo. Und jetzt sag du mir, wie du solchen Leuten verbieten willst, sich zu rächen. (Vojnović 2016, 99)

Im ersten Satz des Zitats formuliert die Figur ein Kriegsverbrecher relativierendes Argument; darauf folgt ein Argumentationsschritt der allgemeinen Verschwörung gegen die Serben - und zwar mit Hilfe von Strategien im Tätergedächtnis, die Assmann mit „Aufrechnen“, „Externalisieren“ und „Umfälschen“ bezeichnet hat (Assmann 2016, 169-182) und die im Roman auf suggestive Weise entpersonalisiert eingebracht werden. Das für dialogisches Erinnern grundsätzlich wichtige Zugeständnis auch des eigenen Leidens wird in der Argumentation der Figur jedoch gezielt zur Legitimation von Rache gebraucht. Erst darauf setzt Danilo nun Nedeljkos persönliche Opfererfahrung: 
„[...] Veš, on nikoga razen tebe in Duše ni imel. Ko so mu vzeli vaju, se mu je zrušil cel svet.“ Nisem vedel, kdo mu naju je vzel, in sumil sem, da je to še ena v nizu zarot proti Srbom in Srbiji [...]. (Vojnović 2013a, 126)

„[...] Weißt du, er hatte ja außer dir und Duša niemanden. Als man ihm euch genommen hat, ist seine ganze Welt zusammengebrochen. "Ich wusste nicht, wer ihm uns beide genommen hatte, und ich vermutete, dass das noch eine in einer Reihe von Verschwörungen gegen die Serben und gegen Serbien war. (Vojnović 2016, 112-113)

Vladan sieht sich selbst also erneut, ohne eigenes Zutun, ins Zentrum eines Opfernarrativs versetzt. Was aber hat es nun mit der Geschichte seines Großvaters Milutin auf sich? Milutins Familie lebte in der Herzegowina und wurde im Zweiten Weltkrieg von der kroatischen Ustaša ermordet. Danilos Erzählung darüber fasst Vladan folgendermaßen zusammen:

Resnico o družini Borojević iz vasi Žilice v okolici Tomislavgrada, ki so jo nekega jutra leta 1942 požgali ustaši [...]. Zaklana in ustreljena trupla [...] so zmetali na nekaj metrov visok mrtvaški kup pred njihovima gorečima hišama, nato pa so dolgo opazovali prizor in uživali v vonju srbskega pepela. Vsaj tako je Danilo povzel pripoved svoje matere, [...]. (Vojnović 2013a, 122)

die Wahrheit über die Familie Borojević aus dem Dorf Žilice in der Nähe von Tomislavgrad [...], das eines Morgens des Jahres 1942 von der Ustascha [...] niedergebrannt wurde. Die abgeschlachteten und erschossenen Körper [...] warfen sie auf einen mehrere Meter hohen Leichenhaufen vor ihren brennenden Häusern, und dann weideten sie sich an dem Anblick und genossen den Geruch der serbischen Asche. So jedenfalls hatte Danilo die Erzählung seiner Mutter zusammengefasst, [...]. (Vojnović 2016, 110)

Welche Folgen hat nun das Anhören von Geschichten über Opfer in der eigenen (Täter-)Familie für den Erzähler?

\section{Die Involviertheit des Zuhörers: Effekte des Opfernarrativs}

Im Text klingt durchaus an, dass die Erzählungen von Emir und Danilo den Protagonisten beeinflussen, allerdings zeigt der ironische Stil, dass Vladan sich dieser Effekte durchaus bewusst wird: „Emirjeve besede so mi namreč zvenele skrivnostno in njegova prijetno leseno dišeča topla soba je ustvarjala vtis ovalne pisarne nekakšne skrivne bratovščine upokojenih oficirjev“ (Vojnović 2013a, 87-88) [„Emirs Worte hatten etwas Konspiratives, und sein warmes Zimmer mit dem angenehmen Holzduft wirkte auf mich wie das Oval Office einer ehemali- 
gen geheimen Brüderschaft pensionierter Offiziere“ (Vojnović 2016, 78)]. Ähnlich spürt Vladan die suggestive Wirkung in Danilos Argumentation: „Na neki čuden način so mi Danilove besede prijale in v sebi sem čutil naraščajočo željo, da bi jim verjel in skril za njimi vse svoje dvome in strahove“ (Vojnović 2013a, 112) [„Auf eine seltsame Weise taten mir Danilos Worte wohl, und ich spürte den Wunsch, ihnen zu glauben und hinter ihnen meine Zweifel und Ängste zu verstecken“ (Vojnović 2016, 99)].

Im unmittelbaren Anschluss an Danilos Erzählung, auf der Rückkehr aus Serbien und unterwegs Richtung Kroatien, imaginiert Vladan das Massaker in dem kroatischen Dorf im Herbst 1991 aus der Perspektive seines selbst leidenden Vaters. Das folgende Zitat zeigt, wie tief der Ich-Erzähler (der von seinem Vater übrigens distanziert als „Nedeljko“ spricht) versucht, sich in die damalige Situation seines Vaters, mithin in eine Täterpsyche, hineinzuversetzen:

Morda je Nedeljko res videl, kako gorijo živi ljudje, kako matere gledajo umiranje lastnih otrok [...], a se ni več zmogel iztrgati začasne neprištevnosti. Utapljal se je v bolečem občutku izigranosti, izdanosti in opeharjenosti in sejal je smrt, da bi kaznoval to življenje, ki mu je uzelo vse, kar je imel rad. (Vojnović 2013a, 126-127)

Vielleicht hat Nedeljko tatsächlich gesehen, wie lebendige Menschen brennen, wie Mütter das Sterben ihrer eigenen Kinder mit ansehen [...], konnte sich aber nicht aus der vorübergehenden Unzurechnungsfähigkeit herausreißen. Versank im schmerzlichen Gefühl, ausgetrickst, verraten, übervorteilt worden zu sein, und säte den Tod, um das Leben zu bestrafen, das ihm alles genommen hatte, was er liebte. (Vojnović 2016, 113)

Dem Protagonisten drängt sich so unter dem Eindruck der Erzählung seines Onkels Danilo eine Parallelisierung der Schicksale des Großvaters und des Vaters auf: der Großvater blickt auf die Leichen seiner Angehörigen in seinem von der kroatischen Ustaša niedergebrannten serbischen Dorf. Er ist Opfer eines Verbrechens. Der Vater blickt auf die Leichen der Bewohner eines kroatischen Dorfes, die auf seinen Befehl hin ermordet wurden. Er ist Täter eines vergleichbaren Verbrechens, eines Rache-Aktes also. Diese Szene plastischer Visualisierung wird in einer späteren Passage gedoppelt, als Vladan Fotos zu Gesicht bekommt, die seinen Vater vor den Leichen der kroatischen Dorfbewohner zeigen, die er ebenfalls auf einen Haufen stapeln ließ, so wie er es selbst aus den Erzählungen über die toten Verwandten seines Vaters Milutin wusste.

Die tiefgehende persönliche Involviertheit des Erzählers in die ambivalente Opfer- und Verbrechergeschichte seiner Familie zeigt sich im Buch später in einem markanten Flashback, auf den ich nun eingehen werde. Zurück in Ljubljana erlebt der Protagonist, wie Jugendliche in einer Bar eine Balkanparty mit Turbofolk-Musik feiern. Der betrunkene Protagonist, dessen Gedanken zwischen seinem Vater und dem unklaren Balkanverständnis der Ljubljaner Jugendlichen 
ruhelos hin- und herpendeln, verliert des lauten Turbofolks wegen schließlich die Nerven. Ohne nachzudenken brüllt er einen Fluch, den früher sein Vater benutzt hat. Unmittelbar nachdem er unbewusst seines Vaters Worte ausgesprochen hat, taucht schlagartig eine mit diesem Fluch verbundene, vergessen geglaubte Kindheitserinnerung wieder auf. Die Erinnerung an einen gemeinsamen Besuch auf dem Pulaer Filmfestival im Amphitheater („Arena“) Ende der 1980er Jahre ist - typisch für traumatische Erinnerungen bzw. Flashbacks (Caruth 1995, 152) unvorhergesehen und außergewöhnlich klar und deutlich:

„More marš u pičku materinu!“ [...] Primože sem dal na „mute“ in se odsotno zazrl predse. Bila je Nedeljkova kletvica, [...] in naenkrat sem ga živo videl, kako se, držeč me čvrsto za roko, prepira s tipom na vhodu v Areno. (Vojnović 2013a, 148)

„More marš u pičku materinu!“ [...] Ich hatte die Primože auf „mute“ geschaltet und starrte jetzt abwesend vor mich hin. Das war Nedeljkos Fluch gewesen, [...] und auf einmal sah ich ihn lebendig vor mir, wie er, mich fest an der Hand haltend, sich mit einem Typ am Eingang zur Arena streitet. (Vojnović 2016, 131)22

Die Auseinandersetzung des Ich-Erzählers mit der Tätergeschichte seines Vaters sowie dem implementierten Opfernarrativ ist also untrennbar mit den verdrängten und grundliegend positiv konnotierten Erinnerungen an die Kindheit in Jugoslawien und an seinen Vater verknüpft. Sind diese Erinnerungen durch den Zerfall Jugoslawiens, den Heimat- und Sprachverlust und den angeblichen Tod des Vaters als traumatisch zu werten, so werden sie nun in der Auseinandersetzung des Erzählers mit seinem Vater in Form von Flashbacks aktiviert und verleihen der Täterfigur des Vaters aus der Perspektive des Sohnes eine ambivalente Zeichnung.

Die letztendliche Konfrontation mit dem untergetauchten Vater in einem Café in Wien ist unversöhnlich. Hier dominiert des Vaters Schlüsselbegriff „Schicksal“, den dieser argumentativ aus dem familiären Opfernarrativ herleitet. Und nun kommt Nedeljko das erste Mal selbst zu Wort:

In potem sem [...] se spomnil zgodbe svojega očeta ... in kupa, na katerem so bila trupla njegove, moje, tvoje družine. [...] Vse je bilo zame že vnaprej določeno. Nikoli tu ni bilo nobene izbire. [...] Tista vojna se je s to vojno le nadaljevala in Milutinova usoda je postala moja usoda. (Vojnović 2013a, 256-257)

22 ,Primož‘ ist eine auf den slowenischen Reformator Primož Trubar anspielende stereotype Bezeichnung für die Slowenen (vgl. Vojnović 2016, 255), von denen sich der Ich-Erzähler hier distanziert. 
Und dann habe ich [...] mich an die Geschichte meines Vaters erinnert ... und an den Haufen, auf dem Leichen seiner, meiner, deiner Familie gelegen haben. [...] Alles war für mich schon im Voraus bestimmt. Es hat da nie eine Wahlmöglichkeit gegeben. [...] Jener Krieg damals hat mit diesem Krieg nur eine Fortsetzung gefunden, und Milutins Schicksal wurde mein Schicksal. (Vojnović 2016, 230-231)

Vladan jedoch ist durch die Auseinandersetzung mit den Erzählungen der Anderen bereits auf das Opfernarrativ vorbereitet, das ihn hier durch die geschickte Reihung der Worte „njegove, moje, tvoje družine“ [seiner, meiner, deiner Familie] erneut mit einverleibt. Er nimmt eine anklagende Rolle ein und eine aktive Dekonstruktion des Spiels seines Vaters „Z izmišljenimi usodami“ (Vojnović 2013a, 257-258) [„,mit den erfundenen Schicksalen“ (Vojnović 2016, 231)] vor.

General Borojević [...] [je] [o]digral ulogo [...] nedolžne žrtve [...]. bez besed [me je] spraševal, zakaj jaz [...] si ne želim niti slišati njegove zgodbe. [...] Zgodbe, ki sem ji tako zlahka nasedel, ko so mi jo skupaj po delčkih pripovedovali Emir, Danilo in Brane [...]. (Vojnović 2013a, 264)

General Borojević [...] hatte die Rolle des [...] unschuldigen Opfers gespielt, [...]. [Er] hatte [...] mich ohne Worte gefragt, weshalb ich [...] seine Geschichte nicht einmal hören wollte. [...] Eine Geschichte, der ich so leicht aufgesessen war, als sie mir Stück für Stück Emir, Danilo und Brane erzählt hatten, [...]. (Vojnović 2016, 238)

\section{Das Leiden der Anderen?}

Zum Schluss wird auf den Titel des Beitrags Bezug zu nehmen sein, um einen Punkt zu erläutern, der bisher nicht direkt zur Sprache kam. Wer sind nun eigentlich die Anderen, deren Leiden den zuhörenden Erzähler umtreiben? Durch die Suche der Erzählerfigur nach dem Vater und nach den Ursachen der Kriegsverbrechen, die dieser im Kroatienkrieg begangen hatte, wird im Roman vordergründig die Täterperspektive und das ambivalente Opfernarrativ akzentuiert, das zur Entlastung des Verbrechens zum Einsatz kommt. Die Täterfigur erfährt im Buch eine durchaus vielschichtige Zeichnung und erhält am Ende auch selbst eine Stimme. In dieser Vater-Sohn-Konstellation agiert Vladan allerdings nicht als empathischer Zuhörer und weigert sich zum Schluss, das Leiden seines Vaters anzuhören und zuhörend dessen Verbrechen zu rehabilitieren: „Ne vem, zakaj si prišel, če me sploh nočeš poslušati. 'Zakaj naj te poslušam?! Vzgojil si me kot ateista, komunjaro jedna. Zdaj bi mi pa pridigal o usodi““ (Vojnović 2013a, 260) [,,Ich weiß nicht, weshalb du gekommen bist, wenn du mir überhaupt nicht 
zuhören willst. 'Warum sollte ich dir zuhören?! Du hast mich zum Atheisten erzogen, du großer Kommunist, Und jetzt möchtest du mir vom Schicksal predigen““ (Vojnović 2016, 234)].

Das dialogische Potenzial des Romans äußert sich also weniger in der Figurenkonstellation, denn Vladans Gesprächspartner sind weit von einer Anerkennung einer Perspektive des Anderen entfernt. Dialogisches Erinnern geschieht auf der Ebene des Diskurses, mithin der Dekonstruktion des Opfernarrativs durch die Hauptfigur. Worin liegt nun das Entscheidende in der Konzeption der zuhörenden Erzählerfigur? Wie oben ausgeführt liegt die Funktion bezeugender Zuhörerschaft in der Übernahme von Verantwortung. ${ }^{23}$ Und genau dies tut Vladan, indem er sich eben nicht vom manipulativ eingesetzten Opfernarrativ vereinnahmen lässt. Er übernimmt Verantwortung für die Anderen, für die während des Massakers im November 1991 getöteten Dorfbewohner, denen im Roman selbst an keiner Stelle eine Stimme oder Perspektive zukommt. Ihre Existenz wird jedoch durch Fotos, die den Vater neben Leichen und vor einem Leichenberg zeigen, sowie in kurzen Imaginationen des Protagonisten immerhin angedeutet:

Šele zdaj sem dojel, da stoji vojak ob ženskem truplu in se s svojo nogo opira nanj, da se mu med zavezovanjem vezalk ne bi bilo treba preveč sklanjati. [...] Na tretji je poleg mrtvaške grmade, na katero je bilo zdaj odvrženo še peto, otroško truplo, stal vojak. (Vojnović 2013a, 234)

Jetzt erst erfasste ich, dass der Soldat neben einem weiblichen Torso stand und seinen Fuß auf ihn gesetzt hatte, um sich beim Binden der Schnürsenkel nicht zu tief bücken zu müssen. [...] Auf dem dritten [Foto] stand der Soldat neben dem Leichenhaufen, auf den jetzt noch ein fünfter, ein Kinderkörper, geworfen war. (Vojnović 2016, 210)

Die Analogie zwischen dem Leichenhaufen der eigenen Familie, von dessen Leiden Vladans Großvater kein Zeugnis abgeben konnte oder durfte, und dem Leichenhaufen der Bewohner des kroatischen Dorfes, deren Ermordung der Vater $\mathrm{zu}$ verantworten hat, wird vom Vater mit Hilfe des Opfernarrativs als Rechtfertigung eines Rache-Aktes gebraucht. Der sich dieser Argumentation verweigernde Protagonist übernimmt somit deutlich Verantwortung für die Vergangenheit.

Trotz - oder gar aufgrund - dieser klaren Positionierung ist es Goran Vojnović gelungen, in seinem Roman Zwischenräume herauszuarbeiten und Alternativen zu binaristischen Vorstellungen von Opfern und Tätern aufzuzeigen. Er nähert sich demnach den von Daniela Mehler geforderten „Graustufen“ an, dem Aufzeigen der „Doppelrollen von Akteuren als Täter-Opfer sowie Opfer-Täter“ $(2015,300)$ als

23 Vgl. das unter Punkt 2 ausgeführte Konzept der sekundären Zeugenschaft. 
Basis für einen dialogischen Erinnerungsdiskurs. Das Besondere am Ansatz des Autors ist es, eine ambivalente Täterfigur zu zeichnen ohne das konkrete Kriegsverbrechen zu bagatellisieren oder zu entschuldigen. Auf der Ebene der literarischen Inszenierung werden also die Prägung des sozialen Gedächtnisses durch Opfernarrative und Verdrängungsstrategien gezeigt, sowie die Schwierigkeiten, aus diesen Familienkonstellationen auszubrechen. Die Wirkung des Romans wiederum, der in der postjugoslawischen Region sehr positiv aufgenommen wurde, zeugt vom Potenzial der Subkultur der postjugoslawischen Literaturszene, ${ }^{24} \mathrm{zu}$ einem dialogischen Erinnerungsdiskurs in der Region beizutragen.

\section{Literaturverzeichnis}

Assmann, Aleida. Der lange Schatten der Vergangenheit: Erinnerungskultur und Geschichtspolitik. 2. Auflage. München: C. H. Beck, 2014.

Assmann, Aleida. Das neue Unbehagen an der Erinnerungskultur: Eine Intervention. 2. Auflage. München: C. H. Beck, 2016.

Baer, Ulrich. Hg. „Niemand zeugt für den Zeugen“: Erinnerungskultur und historische Verantwortung nach der Shoah. Frankfurt a. M.: Suhrkamp, 2000.

Beganović, Davor. „Poštovanje Drugoga, patnja Drugoga“. Književna Istorija 157 (2015): 367380.

Bremer, Alida. „Neuere serbische Literatur: Zwischen Geschichte, Mythos und Nation“. Die Neue Gesellschaft: Frankfurter Hefte 39.5 (1992): 457-462.

Caruth, Cathy. „Recapturing the Past: Introduction“. Trauma: Explorations in Memory. Hg. Cathy Caruth. London: John Hopkins University Press, 1995. 151-157.

Čeh Steger, Jožica. „Gewaltphänomene in der slowenischen Literatur: Die Kriegsgewalt und die Gewissensfrage“. Verbrechen - Fiktion - Vermarktung: Gewalt in den zeitgenössischen slavischen Literaturen. Hg. Laura Burlon, Nina Frieß, Irina Gradinari und Katarzyna Różańska. Potsdam: Universitätsverlag Potsdam, 2013. 197-210.

Djordjević, Mirko. „Die Literatur der populistischen Welle“. Serbiens Weg in den Krieg: Kollektive Erinnerung, nationale Formierung und ideologische Aufrüstung. Hg. Thomas Bremer, Nebojša Popov und Heinz-Günther Stobbe. Berlin: Arno Spitz 1998. 225-241.

Dragović-Soso, Jasna. ,Saviours of the Nation': Serbia's Intellectual Opposition and the Revival of Nationalism. Montreal und Kingston: McGill-Queen's University Press, 2002.

Höpken, Wolfgang. ,Jasenovac - Bleiburg - Kočevski Rog: Erinnerungsorte als Identitätssymbole in (Post-)Jugoslavien“. Geschichte (ge-)brauchen: Literatur und Geschichtskultur im Staatssozialismus. Jugoslavien und Bulgarien. Hg. Angela Richter und Barbara Beyer. Berlin: Frank \& Timme, 2006. 401-429.

24 Vgl. die unter Punkt 1 vorgenommenen Ausführungen und Quellenangaben zum Begriff der postjugoslawischen Literatur. 
Konitzer, Werner. „Opferorientierung und Opferidentifizierung: Überlegungen zu einer begrifflichen Unterscheidung“. Das Unbehagen an der Erinnerung: Wandlungsprozesse im Gedenken an den Holocaust. Hg. Margit Frölich, Ulrike Jureit und Christian Schneider. Frankfurt a. M.: Brandes \& Apsel, 2012. 119-127.

Kowollik, Eva. Geschichte und Narration: Fiktionalisierungsstrategien bei Radoslav Petković, David Albahari und Dragan Velikić. Berlin: LIT, 2013.

Matijević, Tijana. „National, Post-national, Transnational: Is Post-Yugoslav Literature an Arguable or Promising Field of Study?" Grenzräume - Grenzbewegungen: Ergebnisse der Arbeitstreffen des Jungen Forums Slavistische Literaturwissenschaft Basel 2013 / Frankfurt (Oder) und Slubice 2014. Band 1. Hg. Nina Frieß, Gunnar Lenz und Erik Martin. Potsdam: Universitätsverlag Potsdam, 2016. 101-112.

Mehler, Daniela. Serbische Vergangenheitsaufarbeitung: Normwandel und Deutungskämpfe im Umgang mit Kriegsverbrechen, 1991-2012. Bielefeld: transcript, 2015.

Milosavljević, Olivera. „Der Mißbrauch der Autorität der Wissenschaft“. Serbiens Weg in den Krieg: Kollektive Erinnerung, nationale Formierung und ideologische Aufrüstung. $\mathrm{Hg}$. Thomas Bremer, Nebojša Popov und Heinz-Günther Stobbe. Berlin: Arno Spitz 1998. 159-182.

Rothberg, Michael. Multidirectional Memory: Remembering the Holocaust in the Age of Decolonization. Stanford: Stanford University Press, 2009.

Sabrow, Martin. „Held und Opfer: Zum Subjektwandel deutscher Vergangenheitsverständigung im 20. Jahrhundert“. Das Unbehagen an der Erinnerung: Wandlungsprozesse im Gedenken an den Holocaust. Hg. Margit Frölich, Ulrike Jureit und Christian Schneider. Frankfurt a. M.: Brandes \& Apsel, 2012. 37-54.

Schulze Wessel, Martin. „Einleitung“. Opfernarrative: Konkurrenzen und Deutungskämpfe in Deutschland und im östlichen Europa nach dem Zweiten Weltkrieg. Hg. K. Erik Franzen und Martin Schulze Wessel. München: Oldenbourg, 2012. 1-8.

Sundhaussen, Holm. „Serbische Historiographie zwischen nationaler Legitimationswissenschaft und postnationalem Paradigmenwechsel“. Klio ohne Fesseln: Historiographie im östlichen Europa nach dem Zusammenbruch des Kommunismus. Hg. Alojz Ivanišević, Andreas Kappeler, Walter Lukan und Arnold Suppan. Frankfurt a. M.: Peter Lang, 2002. 411-419.

Trgovčević, Ljubinka. „Historiographie in der BR Jugoslawien 1991-2001“. Klio ohne Fesseln: Historiographie im östlichen Europa nach dem Zusammenbruch des Kommunismus. Hg. Alojz Ivanišević, Andreas Kappeler, Walter Lukan und Arnold Suppan. Frankfurt a. M.: Peter Lang, 2002. 397-409.

Vervaet, Stijn. „Intersecting Memories in Post-Yugoslav Fiction: The Yugoslav Wars of the 1990s through the Lens of the Holocaust“. Post-Yugoslav Constellations: Archive, Memory, and Trauma in Contemporary Bosnian, Croatian, and Serbian Literature and Culture. Hg. Stijn Vervaet und Vlad Beronja. Berlin und Boston: De Gruyter, 2016. 99-126.

Vojnović, Goran. Jugoslavija, moja dežela. Ljubljana: Študentska založba, 2013a [2011].

Vojnović, Goran. „Možda Jugoslavija nekome smeta, ali to je moj kulturni prostor“. Interview mit Mladen Pleše. Jutarnji list, 20. September 2013b, https://www.jergovic.com/ppk/mozdajugoslavija-nekome-smeta-ali-to-je-moj-kulturni-prostor/ (11. September 2018).

Vojnović, Goran. Vaters Land. Übers. von Klaus Detlef Olof. Wien und Bozen: Folio, 2016. Welzer, Harald. Das kommunikative Gedächtnis: Eine Theorie der Erinnerung. München: C. H. Beck, 2002. 